\title{
Promotion of the participation of families and the community in educational institutions of basic education
}

Fomento de la participación de las familias y comunidad en instituciones educativas de educación básica

\section{Bladimir Jonny Luján García}

Docente en Educación Secundaria, Universidad César Vallejo, https://orcid.org/0000-0002-5915-6486, blujanga29@ucvvirtual.edu.pe

\section{Revista Iberoamericana de la Educación \\ Vol - Especial 12021 \\ e-ISSN: 2737-632x}

Abstract: The present study aims to identify the promotion of family and community participation in basic education schools and their involvement in various aspects, to this end, a review of the available state of the art was carried out, based on key terminologies and the grouping by categories of this fact, which is evident in other states of the region and the world. It also aims to publicize the results of the analysis carried out. We found that educational policies lack solid guidelines or few efforts are made to promote participation. Likewise, that educational management only encourages basic levels of participation: informative and collaborative. In addition, there is no pedagogical management in place that allows for this involvement in a positive way, despite the recognition of its importance in improving student learning.

Key words: Community participation, Educational management, Family participation, Institutional Leadership 
Resumen: El presente estudio pretende identificar la promoción de la participación familiar y de la comunidad en instituciones educativas de educación básica y su implicancia en diversos aspectos, para ello se realizó una revisión del estado del arte disponible, a partir de terminologías claves y la agrupación por categorías de este hecho, que es constancia en otros Estados de la región y del mundo. Asimismo, tiene como finalidad dar a conocer los resultados del análisis realizado; en ello encontramos que, las políticas educativas carecen de lineamientos sólidos o son pocos los esfuerzos que se hacen en cuanto al fomento de la participación. Igualmente, que la gestión educativa solo fomenta niveles básicos de participación: informativo y colaborativo. Además, que no se está ejerciendo una gestión pedagógica que permita este involucramiento de manera positiva, pese a que se reconoce la importancia que acarrea para lograr mejorar los aprendizajes de los estudiantes.

Palabras clave: Gestión educativa, Liderazgo institucional, Participación de la familia, Participación de la comunidad

\section{INTRODUCTION}

Una de las barreras que divide a un Estado de otro es el producto que logra trienalmente resultado de un Programa para la Evaluación Internacional de Estudiantes, PISA, con el que sale a relucir el posicionamiento de su sistema educativo en eficiencia, equidad y calidad. Asimismo, a estos aspectos se les une la preponderancia del desempeño e idoneidad de los docentes, la eficacia y liderazgo de la dirección de los directivos (Organización para la Cooperación y el Desarrollo Económico (OCDE), 2019; Oficina de Medición de la Calidad de los Aprendizajes (UMC), 2019; Ministerio de Educación del Perú (Minedu), 2014). Por ende, los bajos resultados, la baja calidad y el 
fracaso educativo de un determinado país es imputado a su sistema educativo, aún más, a la gestión y el liderazgo en las escuelas (Ochoa et al., 2021). Debates internacionales actuales contemplan la imperiosa necesidad de considerar dentro de las políticas educativas el fomento de la participación familiar y de la comunidad en las instituciones educativas (Zavala, 2019; Córdova, 2018; Barrientos et al., 2016; Salimbeni, 2015), ello debido a que es una condición necesaria para garantizar una transparente, idónea y democrática gestión escolar, así como ciertas formas de involucramiento que pueden contribuir y tener un impacto positivo sobre los aprendizajes, el fortalecimiento valórico, emocional, personal y el desenvolvimiento en la sociedad del ser humano en formación. Concurre a nivel mundial y en exclusividad en países de bajos ingresos, como los de Latinoamérica, dificultades entorno a la inclusión en educación (Organización de las Naciones Unidas para la Educación, la Ciencia y la Cultura (Unesco), 2020). Un sesgo de ello es la escasa promoción de la participación de las familias y comunidad en los centros educativos. Debido, primero, a que las familias no asumen el compromiso de inmiscuirse en la educación de sus hijos de forma directa en la institución educativa; segundo, la participación social o de la comunidad se encuentra aislada de las escuelas, siendo estos entes descontextualizados o desconectados del contexto situacional de la sociedad (Ovillo, 2019; González, 2019; Rojas, 2017). Por tanto, se infiere que es muy poco lo que hace la gestión escolar en cuanto a entablar estrategias viables de comunicación y participación, y si lo hace, no es de manera consultiva y participativa, sino se da en un nivel de imposición.

En países como el Perú, recientemente desde la época de los 90 la promoción de la participación de la familia cobra singular "importancia" en las políticas educativas, tal es así que la Ley General de Educación, N. 28044 , pone énfasis en la necesidad del fomento del involucramiento de estos en los 
asuntos relacionados con la institución educativa (IE). Esta relevancia tuvo un proceso aletargado, tanto así que recién en el 2016 se establece un Reglamento de Ley $\mathrm{N}^{\mathrm{o}} 28628$ donde se normaliza la inserción de las agrupaciones de las familias en los centros educativos; no obstante, la figura es poco o nada perceptible.

Si bien, dichas normatividades sugieren que la educación, específicamente la básica, tenga fuertes vínculos entre escuela, familia y comunidad, aún se sigue priorizando la participación de las familias a aspectos meramente políticos y/o administrativos, más no centrada en el apoyo del proceso de aprendizaje de los estudiantes dentro de las instituciones educativas y otros aspectos imprescindibles (Ovillo, 2019; Córdova, 2018; Pérez, 2015). Más aún, se ha dejado rezagado la participación de la comunidad local, como aliado estratégico, que permita aportar en la solución de problemáticas sociales, de convivencia y las que se evidencia en los resultados del rendimiento, aun extremadamente bajo, en las evaluaciones nacionales e internacionales.

Es emergente consolidar el aspecto emocional, valorativo y la preparación para el desenvolvimiento en la sociedad de la persona en formación con un acompañamiento en el transcurrir de su etapa escolar (Ovillo, 2019; Tamariz, 2013; Burgoyne y Reynolds,1997). Por ende, se hace necesario la participación familiar y de la comunidad dentro de las escuelas para el logro de este objetivo, más aún, con la finalidad de brindar un desarrollo óptimo de la gestión institucional y alcanzar los objetivos para el progreso y mejora de los aprendizajes (Holguín-Alvarez et al., 2020). De igual forma, es indispensable conceptualizar las palabras clave, directrices del presente artículo. Primero, el término participación de la familia va en relación al 
involucramiento que tiene cualquier miembro del hogar (padres, tíos, abuelos u otros integrantes) en actividades o actos que realiza la IE. Esta implicación hace alusión a que estos agentes educativos realizarán acciones como opinar, proponer, tomar ciertas decisiones y/o disentir en los diversos espacios de la escuela (López y Ramírez, 2020; Unesco, 2004).

Segundo, el término participación de la comunidad está relacionada, en primer lugar, a la inclusión del modo y perspectivas culturales, los conocimientos y las experiencias del entorno o contexto social de la escuela como mecanismos de aprendizaje de los estudiantes. En segundo lugar, a los demás agentes o actores con las que el estudiante convive en la IE (Delgado, 2017; Sorados, 2010; Ministerio de Educación Nacional, 2008). Es decir, el primer apartado va relacionado con la intervención que realizan los agentes externos del centro educativo denominados aliados estratégicos: municipalidad, comisaría, posta de salud, parroquia, organizaciones sociales, entre otros (comunidad local), como una red educativa de apoyo que prestan servicios de colaboración a la IE con la finalidad de lograr los objetivos institucionales que van dirigidos a la mejora de los aprendizajes, todavía más, para contribuir en la inserción e involucramiento del contexto social(Beltran et al., 2021; Cárdenas-Palomino et al., 2021). En el segundo apartado están los demás integrantes de la comunidad escolar: auxiliares de educación, psicólogo educativo, personal administrativo y otros que contribuyen a la educación de los estudiantes.

Por consiguiente, conviene preguntarse cuál es el grado del fomento de la participación familiar y de la comunidad dentro de las instituciones educativas de la educación básica. En particular, en este trabajo pretendemos identificar la promoción de estos dos agentes educativos en las escuelas y su implicancia en diversos aspectos de los citados espacios de aprendizaje. Si bien, la presente investigación tiene la característica descriptiva, discurrimos 
que ofrece un panorama exhaustivo e interesante para permitir nuevas investigaciones.

\section{MATERIALS AND METHODS}

Se realizó una revisión exhaustiva y sistemática del estado del arte y literatura actual sobre el tópico, para ello se recurrió a términos clave de búsqueda: participación de la familia, participación familiar, participación de la comunidad, participación comunitaria (todo ello relacionado con la escuela) y cruzadas con: participación social, gestión educativa, gestión institucional, gestión comunitaria, gestión pedagógica, liderazgo directivo, liderazgo pedagógico, inclusión educativa, relación escuela y familia, con sus

respectivas traducciones al inglés. Asimismo, se recurrió a motores de búsqueda los índices de: Google Scholar, Redalyc, Scielo, Latindex, Springer Link, Dialnet, Doaj, Base, Jstor, EbscoHostScopus y repositorios institucionales.

Para tal hecho, se tomaron en cuenta producciones e investigaciones difundidas en revistas y repositorios arbitrados, secciones y libros completos producidos por expertos en el tema, como también informes o reportes de organismos internacionales. Se delimitó como periodo principal de búsqueda la temporalidad 2016 - 2020; aunque, igualmente fue necesario recurrir a estudios anteriores para fortalecer la argumentación respectiva.

\section{RESULTS}

Se localizaron en general más de 75 artículos e investigaciones relacionados al tema, de los cuales fueron tomados en cuenta 55 de ellos por tener vínculo directo al propósito de indagación. Una vez escogido los recursos pertinentes, tomando en cuenta las principales conclusiones y discusiones de todos los 
estudios seleccionados, se realizó un análisis crítico y reflexivo acerca del fomento de la participación familiar y de la comunidad dentro de las instituciones educativas de la educación básica, tanto para involucrarlos en el mejoramiento de los aprendizajes de los estudiantes y en la participación de una gestión escolar compartida.

En tal dirección, los resultados se agruparon en tres categorías principales y cada una de ellas en subcategorías que forman parte de los apartados del presente artículo. El primer apartado general con los resultados sobre las políticas educativas en torno a la participación familiar y de la comunidad, y divididos en dos secciones específicas: a) La inclusión en relación a la participación y b) Niveles, tipos y formas de participación. El segundo apartado general con los resultados sobre los procesos de gestión para el fomento de la participación de las familias y comunidad, y divididos en tres secciones específicas: a) Valoración del fomento de la participación, b) Liderazgo directivo y pedagógico en el fomento de la participación, y c) Empleabilidad de la tecnología para la participación. El tercer apartado general con los resultados sobre la participación familiar y de la comunidad para la mejora de los aprendizajes, y dividido en una sección específica: Comunidades y proyectos de aprendizaje para la participación. En la Figura 1, se muestran las categorías y subcategorías encontradas: 


\section{Figura 1}

Categorías y subcategorías del fomento de la participación de las familias y de la comunidad en las instituciones educativas

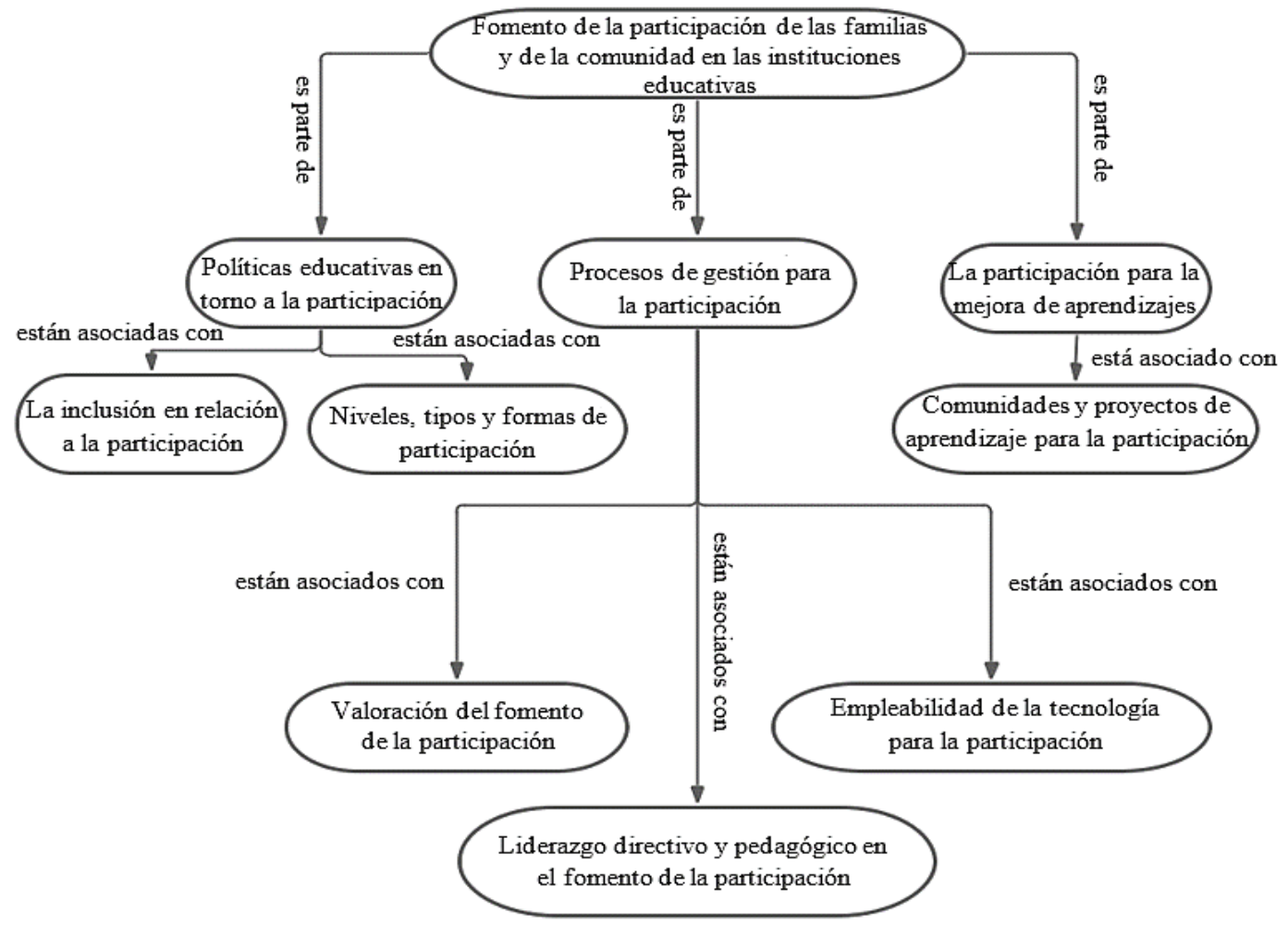

\section{DISCUSSION}

En relación a los resultados se recogen varios aspectos importantes, se reflexiona con base a la forma en la que en la actualidad los sistemas educativos no ponen énfasis al involucramiento más certero de las familias y 
la comunidad dentro de las instituciones educativas, con la finalidad de enriquecer los estudios anteriormente analizados y aportar nuevas hipótesis.

\section{Políticas educativas en torno a la participación familiar y de la comunidad}

Muchas de las investigaciones coinciden que la mayoría de las políticas educativas carecen de lineamientos sólidos o son pocos los esfuerzos que se hacen en cuanto al fomento de la participación del total de las familias y la comunidad dentro de las instituciones educativas (Sucari et al., 2020; Acuña, 2016). Se menciona que existe un ausentismo de acciones y estrategias formativas para las familias que partan desde ellas, debido a que se centran en la exigencia de resultados académicos; más aún, que varias veces la evolución legislativa paraliza lo poco que en normatividades se hace por la promoción de estos agentes educativos en cuanto a derechos de participación en diferentes ámbitos e instancias institucionales (Payà y Tormo, 2016). El tratamiento de este tipo de obligaciones lo único que fomenta es que los centros educativos dirijan su mirada para el logro de los aprendizajes en un solo agente educativo, el docente, dejando rezagado a los otros actores, siendo ellos también piezas claves e impulsores de acciones autoformativas en los estudiantes.

De modo que, se concuerda que es necesario dar una mirada más profunda en cuanto a las políticas educativas de promoción de participación de estos elementos esenciales (familia y comunidad) en la escuela. Para ello, se debe realizar un análisis de las barreras estructurales que impiden este accionar (legislación y roles) y diseñar políticas, más acciones específicas en favor de la importancia e implicación de la participación familiar y de la comunidad en aras del éxito de los aprendizajes de los estudiantes, la labor eficaz de los 
profesionales en educación y la buena administración de estos espacios de aprendizaje (Gaitán et al., 2016; Garreta, 2016).

Dentro de estos cambios y visiones se indica que es urgente también políticas educativas centradas en la formación para la participación no solo del magisterio, agentes poco a nada preparados para implicar a las familias y a la comunidad en sus prácticas, sino de la misma familia y comunidad (López y Ramírez, 2020; Andrés y Giró, 2016a; Vigo et al.; 2016). Esta nueva política en el sistema educativo sería una de las claves para sedimentar el rol y compromiso para una participación activa, vinculante, comprometida y como proceso de retroalimentación para una mejora continua institucional entre todos los actores educativos.

Asimismo, coinciden en que dentro de las políticas educativas se debe fomentar las habilidades comunicativas de las familias, programas de formación familiar centrada en valores y una sensibilización por la participación en la comunidad educativa (López y Ramírez, 2020; Vigo et al., 2016), por medio de una intervención multicompetente que recaiga en el tratamiento de la problemática institucional, de las habilidades sociales y de comunicación, con tareas y actividades auténticas y/o situaciones desafiantes que respondan al involucramiento y brinden respuesta a las dificultades reales del contexto. Por ese motivo, todo sistema educativo de inserción y participación no debe escapar del contexto real, es decir, deben estar enmarcadas a partir de la comprensión integral y sistemática de la realidad, considerando creencias e intereses que influyen en estos agentes (Manzon et al., 2015). 


\section{La inclusión en relación a la participación familiar y de la comunidad en} escuelas.

Dentro de las investigaciones analizadas concurre un tópico recurrente en cuanto a la inclusión como enfoque de derecho, incluso en referencia a una educación de calidad e integradora (Aguiar et al., 2020). Se menciona que existen evidencias científicas para determinar que la participación de todas las familias, así como de la comunidad es esencial para la reducción de situaciones de desigualdad social y educativa; del mismo modo, con el fin de mejorar los logros de aprendizajes de los estudiantes (Arostegui et al., 2016; Calvo y Antonio, 2016). De modo que, una escuela inclusiva, tácitamente, es una escuela participativa donde se incluye a todos los agentes educativos, entre ellos la familia y la comunidad.

Para promocionar la participación como aspecto de inserción en las instituciones educativas es necesario la generación de estrategias y acciones inclusivas. Las investigaciones analizadas, dan como ejemplo de ello, el fomento de las tertulias dialógicas familiares, la generación de comisiones mixtas, comunidades inclusivas, el diseño de proyectos y/o actividades de innovación con proyección de inclusión familiar y comunitaria, con la finalidad de generar relaciones de calidad, respeto, lazos de confianza, comunicación, apoyo y participación (Aguiar et al., 2020; Arostegui et al., 2016; Calvo y Antonio, 2016). El fin último para una escuela participativa, por consiguiente, inclusiva es lograr políticas y prácticas con una cultura de inclusión, con el fin de determinar una repercusión positiva en la mejora de los aprendizajes y otros aspectos de formación del estudiante. 


\section{Niveles, tipos y formas de participación de las familias y comunidad en las escuelas.}

En cuanto a los niveles más comunes de participación de las familias y comunidad en las instituciones educativas identificados en las investigaciones en análisis, encontramos a la participación de corte informativo y colaborativo. Se menciona que las escuelas ofrecen la participación de estos dos actores en estos niveles, pero a partir de una necesidad surgida (Dove et al.,2018; Santana y Reininger; 2018; Pérez, 2015). En esta línea, las actividades de participación están concentradas más que nada en acciones extraeducativas, quiere decir, como los que proveen de recursos y brindan mano de obra, correspondiendo a la atención y el llamado que realizan los docentes y la gestión escolar (Jiménez y Kreisel, 2018). De modo que, se cae en cuenta que los niveles de participación que oferta la escuela surgen desde el planteamiento de su necesidad, y no al contrario.

Un tipo de involucramiento familiar y comunitario recurrente en los trabajos de estudio es de la participación pasiva, indirecta y obligada. Se indica que, al surgir toda propuesta de participación desde la necesidad de la gestión escolar son las familias que sienten coerción y obligación (Villogas, 2019; Santana y Reininger; 2017). Se puede inferir que esta necesidad de participación nace como respuesta a las políticas educativas que exigen que la familia cumpla el rol, por ejemplo, de veedor y controlador de gastos de la administración educativa (Jiménez y Kreisel, 2018). 
Se puede evidenciar además un tipo de participación con correlatividad a lo mencionado anteriormente, la participación en cargos de gestión y representatividad, que igualmente forma parte del cumplimiento de la normatividad educativa. Este involucramiento se da por ejemplo en la elección de su representante al Consejo Educativo Institucional (CONEI), así como del que preside los organismos referidos a la Asociación de Padres de Familia (APAFA), las Asociaciones de Madres y Padres de Alumnos (AMPA), entre otros (Villogas, 2019; Payà y Tormo, 2016). Por lo tanto, hablamos de un injerencia de participación familiar y comunitaria que se limita netamente al aspecto de gestión administrativa y que responde a una política educacional con responsabilidades acortadas a la colaboración o vigilancia. Igualmente, en los estudios en mención se puede apreciar que otras de las formas de participación de la familia y comunidad es la participación en relación a problemáticas de violencia o dificultades suscitadas en la escuela (James y Guzmán, 2016). 
Ello significa que el grado de participación de los padres y también de los aliados estratégicos de la comunidad o sociedad es mayor cuando hay signos de maltrato y/o violencia en los centros educativos. Si bien, en los resultados anteriores trasluce una participación colectiva, la mayoría de las investigaciones denotan que las familias ejercen casi siempre una participación individualizada (García et al., 2016). Ello quiere decir que, la comunicación que entablan con los docentes y la IE es propia por interés de lo que le sucede a su hijo/a, y son ellas quienes deciden de qué manera y en qué momento participar dentro de las posibilidades y limitaciones que la escuela les brinda (Stefanski et al., 2016). Por lo que, en ellas se aboga por un tipo de participación más autónoma o autogestionada, en la que se les permita mayor involucramiento; más incluso considerarlas en la toma de decisiones del quehacer educativo y de la vida institucional para que sean quienes planteen nuevas formas, tipos y estrategias de participación, muy a parte de su ya involucramiento formal en las asociaciones (Santana y Reininger, 2017; Barrientos et al., 2016). 
De igual manera, en el análisis se puede evidenciar una participación pedagógica; no obstante, su recurrencia es en un menor grado, más que nada en niveles como el primario y secundario (Sucari et al., 2020, Akbarul, 2018, Stefanski et al., 2016). Se menciona que el nivel académico de los padres determinada la calidad de participación, como soporte en los procesos educativos (Medina, 2016). También se recoge que la carencia de participación de las familias, en este tipo, se debe a que los docentes no muestran la mejor predisposición para que intervenga en su labor pedagógica dentro de las aulas, pese a que la implicancia de la familia es la más importante y necesaria, debido a que permite el logro y desarrollo de las competencias básicas propuestas por el sistema educativo y fortalece esta práctica con orientaciones formativas (Díaz, 2015; Barrientos et al., 2016; Madrid et al., 2019; Kankaew et al., 2021). Por consiguiente, una participación pedagógica de la familia, inclusive de la comunidad, pese a que no se les considera, es la más efectiva para poder alcanzar las metas y logros de aprendizaje de los estudiantes.

\section{Procesos de gestión para el fomento de la participación de las familias y comunidad}

En las investigaciones revisadas, se puede constatar que existe aún en las escuelas una gestión institucional unidireccional con respecto a la participación familiar y de la comunidad, quiere decir que, los directivos van construyendo una perspectiva de estos dos agentes desde sus propias creencias o idearios, inclusive, descontextualizados, que no responden al contexto real (Santana y Reininger, 2017). Consecuentemente, con lo evidenciado anteriormente, la autoridad de la escuela en su gestión solo los hace participar, en mayor grado, de un nivel informativo, nivel básico de participación (Ramos, 2016). 
Asimismo, los estudios manifiestan que la influencia de la participación de las familias y la comunidad es eficiente en la gestión educativa (Ramos, 2016); por ello, se cae en cuenta que juega mucho el nivel, el tipo y los factores del fomento de la participación en la gestión. De igual forma, se hace hincapié que son ellas las que tienen un rol importante en el desempeño y puesta en marcha de una real gestión escolar (Ünlücan et al., 2017), más aún, en la gestión pedagógica, ya que otorgarán una mirada, sentido y cambio a la gestión directiva, concediéndole un vínculo más directo, un sentido educativo, a través de acciones formadoras que involucre el logro de los aprendizajes y se escape de la mera ejecución administrativa y burocrática de la dirección (Barrientos et al., 2016). 
De la misma manera, pese a que las legislaciones otorgan algunas funcionalidades a las familias en las escuelas, son pocas las acciones que realiza la gestión educativa para promocionar su participación (Garreta, 2016); por ello, en los estudios también se aboga por una gestión democrática - participativa, el cual debe ser abierta, dinámica, integradora, descentralizada e instrumental (Manayay, 2019). Igualmente, se hace hincapié de que la gestión institucional real e idónea tiene como exigencia la participación de todos los actores o agentes educativos: docentes, personal no docente, directivos, padres de familia, en otros términos, comunidad educativa y local en general (Gaitán et al., 2016). Partiendo de lo ya citado, la mayoría de las investigaciones también caen en cuenta que una gestión escolar de calidad y con participación activa conlleva una reorganización de la escuela con implicancia, inclusive, de la comunidad local para el fomento de conexiones ecuánimes entre estos tres actores, familia-comunidad-escuela, con el fin de beneficiar en el aprendizaje de los estudiantes y fortalecer las alianzas del centro educativo y su entorno en pro de lograr una educación de calidad e integral (Epstein y Sheldon, 2016; Alanya Beltrán, 2017; Rojas, 2017), a partir del aprovechamiento de los recursos existentes en ella y propiciando el aprender desde la comunidad, invitación de miembros de la comunidad a compartir experiencias y conocimientos en la institución educativa, (Arostegui et al., 2016). 
Como se mencionó, si bien la escuela da apertura en cierta medida a la participación de las familias (APAFA, AMPA y otras asociaciones), todavía es escasa las estrategias para el fomento de la participación social, es decir, de la sociedad o la comunidad local en la vida académica; por lo cual, surge la necesidad de trabajar por una participación de esta índole, a partir de propuestas redimensionadas, donde se actualice sus funciones, atribuciones y ampliando la representatividad en la misma para lograr una mejor gestión educativa, con transparencia, autonomía y sobre todo encaminado a formar un mejor ciudadano apto para convivir en democracia y en una sociedad justa (Gómez y Vera, 2019; Perales y Escobedo, 2016).

Lo anteriormente señalado conlleva a dar una visión que la escuela es un corpus con características particulares, donde se inmiscuye lo sociocultural, económico y se impregna un carácter político e histórico netamente de su contexto, logrando una relación de carácter netamente democrático y participativo (Barrientos et al., 2016). Quiere decir que las acciones no parten únicamente de la IE, sino de la movilización de la familia en actos de comunión a partir de estrategias y prácticas, donde se consideren las necesidades, como también las expectativas que hagan posible lo mencionado, con el objeto de realizar una mejora de la calidad de la gestión escolar y convertirla en participativa (Ünlücan et al., 2017). En consecuencia, se tendrá una gestión educativa democrática cuando se involucre a la comunidad educativa en general, sin dejar de lado también a la comunidad local. 
En consecuencia, señalan, tanto el proceso de aprendizaje como la vida escolar deben convertirse en la vía para permitir la inclusión más amplia de participación y contribución de la comunidad local, puesto que, aparte de favorecer en los aprendizajes e interacciones de los estudiantes, debido a la presencia de personas de una variada diversidad cultural y social, coadyuva en el apoyo para los trabajadores de la institución educativa y fomenta el aprendizaje e implicación de las familias (Arostegui et al., 2016). Igualmente, una gestión escolar, indican los estudios, que propicie toda acción o estrategia para la participación en la gestión comunitaria, permite el éxito e incremento de la participación social dentro de las escuelas, y esta se debe a que existe un involucramiento en su tarea educativa (Gómez y Vera, 2019; Jacay, 2018). 


\section{Valoración de la participación familiar y de la comunidad en los centros} educativos.

Las investigaciones dan cuenta que la participación del total de las familias en los centros educativos está en un nivel inadecuado o con presencia de falencias (Lavid, 2019; Alves, 2017), así como, en un nivel medio y regular en lo concerniente a la gestión educativa (Zavala, 2019; Ovillo, 2019; Ochoa, 2018). Igualmente, se muestra que existe una relación significativa y una influencia entre la participación de la familia en los diferentes tipos de gestión que ejerce la escuela (An, et al, 2019; Lavid, 2019, Villogas, 2019; Alves, 2017). Del mismo modo, se puede evidenciar que dentro de las influencias de participación están la disposición, la información que reciben, el tipo de comunicación que se entabla, el rol de apoyo pedagógico que se ejerce en casa para fortalecer el aprendizaje adquirido, la cooperación y el voluntariado. Al igual que la participación familiar, los estudios muestran que la participación de la comunidad escolar y local en los diversos tipos de gestión se encuentra en un nivel nulo, bajo o regular (Gómez y Vera, 2019; Apaza, 2018; Delgado, 2017). 
Las investigaciones dan cuenta que el nivel regular de participación se da a través de la intervención en asambleas generales, juntas directivas, comités de aula, representatividad en el CONEI, participación en concursos educativos (Delgado, 2017), implementación de estrategias de participación social en aspectos de convivencia escolar y espacios de cooperación (Gómez y Vera, 2019). De igual manera, indican que comprender el porqué del fracaso o ausencia minoritaria de participación familiar y comunitaria dentro de los centros educativos, debe ser visto a partir de dos ópticas: familia y escuela; en muchos de ellos se concuerda que ambos son causantes de este quiebre en la relación; empero, la primera responsabiliza a la escuela, debido a la falta de un compromiso para promocionar la participación y generar demandas insatisfechas; la segunda responsabiliza a la familia, dado que no existe un compromiso en la cooperación de los procesos de aprendizajes de sus hijos (Madrid et al., 2019). No obstante, existe una contradicción, en razón de que la escuela es consciente del deber de cada familia en el éxito de los aprendizajes (Vigo et al., 2016); sin embargo, tiende a caracterizar la participación de las familias como agentes controladores o fiscalizadores más no como colaboradores de la gestión, manteniéndolos al margen de todas las acciones realizadas (Ramos, 2016; James y Guzmán, 2016). 
Inclusive, los investigadores caen en cuenta que la participación de las familias no precisamente tiene vinculación con el nivel de estudio o los recursos que dispone en el hogar (García et al., 2016), como muchas veces la escuela lo relaciona. Sino también cuenta el nivel de motivación, confianza y seguridad que puedan percibir (Medina, 2016), de la forma como la escuela entiende el hecho de participación, puesto que mayormente se toma en cuenta la participación familiar a un aspecto financista con el fin de apalear las necesidades surgidas, alejándosele ampliamente del sentido actual, acompañar en el aprendizaje y entablar una cultura de democratización de la existencia institucional, impidiendo así la reestructuración del conocimiento surgido en la comunidad y sus epistemes (Jiménez y Kreisel, 2018).

Como se evidenció en apartados anteriores, la participación de las familias en un mayor grado se centra en la representatividad dentro de la gestión administrativa (APAFA, AMPA, entre otros); no obstante, los estudios traslucen la persistencia de la problemática a lo no participación. Estas mismas asociaciones deben lidiar con el involucramiento de las familias en la asociación a su gremio, pese al esfuerzo de la práctica de una gestión democrática (García et al., 2016). 
En consecuencia, se ha podido identificar que existe aún un divorcio tácito de mutuo acuerdo entre la familia y la escuela en cuanto al vínculo de participación en los procesos educativos (James y Guzmán, 2016). Una por su parte inmersa en sus responsabilidades laborales y de índole familiar, y la otra centrada en un trabajo individualizado y apartado de todo vínculo relacionado al contexto del estudiante, pero sí que busca a conveniencia de algunos aspectos para la participación de las primeros, construyendo de esta manera una brecha que los separa grandemente. Por ello, el abismo persiste debido que no se logra calar el real sentido e implicancia del término "participación", solo algunos aspectos de este proceso se asumen como válidas, y otras son ignoradas, más aun marginadas (Madrid et al., 2019), cayendo en cuenta que tampoco se entiende el rol de cada actor y las prácticas que debe asumir en los espacios de aprendizaje.

De igual forma, se indica que existe una necesidad de lograr fortalecer la relación familia - escuela en muchas disciplinas asociadas o directamente relacionadas con la educación, debido a que se reconoce que prevé de beneficios como el desarrollar el aspecto sociocultural y recursos para el mejoramiento de los aprendizajes de los escolares (Pereira y Rivas, 2019, Loaiza, 2017); en esta línea, es menester que la escuela reconfigure su rol educador alcanzando también a la familia. Parte de esta reconversión es que se viene evidenciando en investigaciones más actuales, cambios en las escuelas con respecto a la participación como parte del proceso de una educación inclusiva (Aguiar, 2020), ello debido a algunos avances en los niveles legislativo, de gestión y organización educativa que "exige" buscar espacios de fomento e integración de las familias, además de los trabajos para el cambio de conducta de los agentes educativos (Madrid et al., 2019). 
En síntesis, a medida que se dé cabida a la participación familiar, se incrementa la gestión educativa (Zavala, 2019). Por ello, las escuelas deben desarrollar proyectos educativos articulados, multicompetentes y sensibilizadores que partan del interés y características de la familia, impulsen y faciliten su participación. Igualmente, se debe aperturar espacios para el fomento de una comunicación bidireccional: familia - escuela y viceversa, cooperar con el apoyo del proceso de fortalecimiento de aprendizajes en el hogar, más aun, fomentar y desarrollar habilidades comunicativas y sociales (López y Ramírez, 2020; Vigo et al., 2016).

\section{El liderazgo en el fomento de la participación familiar y de la comunidad} en la escuela.

Muchos de estos estudios determinan que existe correlatividad entre el liderazgo del directivo con la participación familiar y de la comunidad (Madrid, et al., 2019; Córdova, 2018; Andrés y Giró, 2016b). Ello quiere decir que, mientras sea mejor el liderazgo del director, mucho mejor será la participación tanto de la familia y de la comunidad en la IE. Por tanto, se infiere que la falencia en la gestión educativa se debe también a que no se está ejerciendo un liderazgo a nivel de dirección que permita este involucramiento en el contexto educativo de manera positiva (Lavid, 2019; Jacay, 2018). 
Incluso, se menciona que en tiempos actuales existen normatividades educativas exclusivas para los líderes pedagógicos que estipulan la organización de su gestión y establecen sus funciones de hacer participar a las familias en la instituciones educativas (Sucari et al., 2020); de igual manera que conocen y se encuentran empapados de estos lineamientos y la implicancia beneficiosa que acarrea, llegando muchas veces a realizar esfuerzos para fomentarla, pero a partir de estilos de promoción tradicionalistas y restrictivas que no logran certeramente el cometido buscado (Sucari et al., 2020; Barrientos et al., 2016). Adicionando a esta dificultad del directivo, se suman factores contextuales que condicionan la nula o escasa participación (Barrientos et al., 2016).

Además de la desmotivación, desconfianza y ausencia del perfil de los docentes en entablar de manera consciente una articulación de su práctica pedagógica con la familia y comunidad, volviéndolo un elemento complejo de inclusión (Vielma, 2020; James y Guzmán, 2016), cuando ellos fácilmente pueden contribuir en el mejoramiento de la participación familiar desde las aulas con estrategias de intercambio de cultura y valores, acrecentando la comunicación bidireccional y sumando al éxito escolar el logro de los aprendizajes (Vigo et al., 2016). Entendido ello, las familias se transforman en el cimiento que se complementará con el liderazgo pedagógico, tanto del docente y directivo, para impregnarle de un significado diferente a la gestión educativa. Puesto que, a través de la organización escolar y la unión de sus principales agentes se robustecerá la participación (Vielma, 2020). 
En consecuencia, cae en manos y bajo responsabilidad de los dos líderes principales de la escuela (directivos y docentes) entablar y fomentar una relación sólida con la familia y la comunidad, a pesar del carácter heterogéneo y diverso de estos últimos (Andrés y Giró, 2016a; Andrés y Giró, 2016b; Stefanski, 2016). Para ello, es urgente fortalecer el perfil y el rol para el fomento de la participación, en causado en proyectos con una misma dirección, que permita alcanzar el éxito de la fidelización del actuar de la familia en la escuela en el proceso educativo de los estudiantes (Barrientos et al., 2016; Epstein y Sheldon, 2016; Medina, 2016). En caso no se efectuase esta transformación, tampoco se tendrá avances significativos en el desarrollo del aspecto social y comunitario que todo centro educativo debe propiciar (Lavid, 2019). 


\section{La tecnología en el fomento de la participación de la familia y comunidad} en las escuelas.

Como se viene evidenciando, existe notoriamente dificultades en la gestión escolar para la promoción de la participación familiar y comunidad, indiscutiblemente uno de los factores son la carencia o debilitados canales comunicativos que fortalezcan la relación y confianza (Barrientos et al., 2016). Por lo cual, es indispensable recurrir a la tecnología como medio facilitador de este proceso. Varios de los resultados de los estudios evidencian que la tecnología ha realizado inconmensurables aportes a la gestión educacional en cuanto al fomento de la participación familiar y comunidad y a la gestión pedagógica, incrementando el involucramiento de la familia al proceso de acompañamiento en el aprendizaje, proceso tan anhelado por los agentes educativos de la escuela (Londoño, 2017; Ünlücan et al., 2017; Panduro-Ramirez et al., 2021; Quispe et al., 2021). Por esa razón, es necesario la incentivación del empleo responsable y seguro de las herramientas digitales, por medio de programas definidos, ya que su uso adecuado, como se mencionó, operaría también como una de las maniobras educativas de fomento de participación, comunicación, inclusión, acercamiento y actuar pedagógico de la familia y comunidad, así como la potenciación de un aprendizaje de calidad, a partir del aprovechamiento masivo de estos recursos de la sociedad de la información (Chiquillo y Llopis, 2020; López y Ramírez, 2020; González, 2019; Ünlücan et al., 2017). 


\section{Participación familiar y de la comunidad para la mejora de los aprendizajes}

A lo largo del desarrollo de cada apartado, ha traslucido implícitamente que la participación, colaboración, acompañamiento e involucramiento familiar y de la comunidad influye inmensamente en el proceso para mejorar los aprendizajes de los escolares (Akbarul, 2018), más aun, que existe una relación directa y muy significativa con las actividades escolares, el éxito académico, la reducción de deserción o fracaso escolar y la mejora en conjunto del sistema educativo (Andrés y Giró, 2016a; Vigo et al., 2016). Sin embargo, todavía no existe esa confluencia de ambos agentes para lograr este ambicionado propósito (Pereira y Rivas, 2019). Los investigadores de los estudios analizados aconsejan que la participación de la familia debe ser una tarea importante para el complemento del aprendizaje que los estudiantes adquieren en la institución educativa (Manzon et al., 2015).

Por consiguiente, para lograr la calidad y mejora de estos, más aún, instaurar una relación y gestión pedagógica con presencia de la familia y comunidad en la escuela, que es el anhelo de la mayoría de los actores educativos en la actualidad, se demanda la creación y consolidación de nuevos mecanismos de consenso que estén direccionados al empoderamiento, concientización, así como la comprensión que todo cambio o modificación pedagógica se tiene que establecer a partir de estos dos actores con aras a instituir nuevos mecanismos de participación y poder responder a la demanda real (López y Ramírez, 2020; Gaitán et al., 2016). 
A partir de lo manifestado, es tarea de los líderes pedagógicos empezar la creación mancomunada de los mecanismos en mención con las características definidas, empero incluyendo a todos los agentes educativos para su realización (Pereira y Rivas, 2019), con el fin de considerar sus necesidades reales y entablar un compromiso que nazca de sus propias reflexiones. Es decir, cuando la escuela conjuntamente con la familia y comunidad planifican y proponen implementar programas colegiados, pueden transformar patrones que limitaban la participación y generar compromisos en la educación de sus hijos (Epstein y Sheldon, 2016).

\section{Comunidades y proyectos de aprendizaje para el fomento de la participación familiar y comunidad en escuelas.}

Como se demostró en el análisis, a lo largo de la presente investigación, el diseño participativo de estrategias conlleva a grandes mejoras y al logro de los propósitos. Por este motivo las instituciones educativas deben canalizar la construcción de redes de participación para este fin (Dove et al.,2018; Simón et al., 2016), debido a que las asociaciones de trabajo son formas de colaboración significativa que incluye al compromiso de todos (Aguiar et al., 2020; Epstein y Sheldon, 2016). Para ello, se debe iniciar con criterios básicos de formación y capacitación, continuar con acciones perennes de motivación, estímulos, comunicación y un solidario trabajo en equipo donde se comparta el mismo espíritu (Medina, 2016). 
Los estudios indican que parte de estos mecanismos y propuestas de implicación de las familias, así como de la comunidad escolar y local en el contexto de la escuela son las comunidades de aprendizaje (González, 2019; Calvo y Antonio, 2016; Payà, 2016), en vista de que ofrecen un aprendizaje dialógico a través de una articulación con la sociedad de la información y del conocimiento, ejerciendo una educación de calidad por medio de la materialización de la participación e institución de formas nuevas de colaboración.

Parte de la acción mencionada es la creación de programas y proyectos de aprendizaje, grupo de voluntariado, equipos interactivos y dinámicos, aún más, actividades innovadoras donde se trabajen y desarrollen habilidades de diálogo, emocionales y sociales, así como la promoción de la formación en orientación a mejorar la comunicación, confianza, respeto, colaboración mancomunada, el tiempo compartido en tareas escolares y extra escolares de los estudiantes (López y Ramírez, 2020; Medina, 2016; Álvarez, 2016). Es necesario por ello conocer las competencias y habilidades de los actores educativos para aprovecharlas a partir de convenientes procesos participativos de gestión escolar. 
Si bien, el diseño de proyectos de aprendizaje está establecido en el marco normativo, designándole características de colaboración y participación, los estudios señalan que en el ejercicio no se da cumplimiento debido a que las familias se sienten limitadas e incompetentes (Sucari et al., 2020; Calvo y Antonio, 2016). Por consiguiente, las comunidades de aprendizaje deben diseñar proyectos contextualizados a la realidad de la familia y comunidad con características de inclusión, intervención y orientados al logro de la participación e involucramiento (González, 2019; Londoño, 2017, Simón et al., 2016), para ello se puede aprovechar la variedad de herramientas tecnológicas y plataformas virtuales con la finalidad de implicar a todos.

Los beneficios del fomento de la implantación de comunidades de aprendizaje y su implicancia, concluyen los estudios, que fomenta en índices altos la participación de la comunidad, permite la apertura de vías y formas democráticas de implicación tanto en la gestión educativa, institucional y pedagógica; además, concede que más individuos accedan a los espacios de la escuela, en exclusividad de las aulas; también cooperen en las actividades escolares; de igual modo, acrecienta el voluntariado y permite fortalecer prácticas existentes y generar otras más participativas e innovadoras; de igual forma, se fideliza con mayor compromiso el involucramiento de las familias y aliados pedagógicos que ya participaban en la institución educativa; así como se mejora los aprendizajes y la dinámica interna de la escuela (Payà, 2016; Álvarez, 2016). 


\section{CONCLUSIONS}

El fomento o promoción de la participación de la totalidad de los actores educativos, es más de las familias y de la comunidad, cuya inserción es aún débil o poco notoria dentro de las escuelas, es necesario e imprescindible para el mejoramiento de los aprendizajes y desarrollar el aspecto sociocultural en los estudiantes. Por ello, se deben realizar esfuerzos denodados en conseguir este involucramiento a partir de diversas estrategias que surja de la concertación de todos los agentes implicados. Para eso, son emergentes políticas educativas de formación para la participación activa, comprometida, con acciones inclusivas y centrada en valores, que no sean ajenas al entorno situacional, cultural y social, exclusivamente de estos dos agentes.

Del mismo modo, desde la gestión educativa, es necesario implantar niveles, tipos y formas de participación más allá del corte informativo y colaborativo (niveles base de las exigencias de muchas de las normatividades vigentes), que se encauce desde la demanda de la familia y comunidad, más no contrariamente; por esta razón, se debe abogar por una participación autogestionada o autónoma, enmarcada para una participación pedagógica. Por tal razón, debe ser de exigencia una dirección que propicie una gestión institucional, administrativa y pedagógica participativa - democrática, que busque también el involucramiento social y comunitario a través del fomento de habilidades comunicativas y sociales bidireccionales. 
Igualmente, es trascendental el fortalecimiento del rol y perfil de los líderes de la escuela (directivo y docentes) para el fomento de la participación, y este aspecto sea visto como un accionar necesario y complementario para su práctica directiva y pedagógica. Además, el aprovechamiento de los recursos tecnológicos, como canales de comunicación y acercamiento a estos dos agentes para su inserción, deben ser valorados y de gran necesidad emplearlos.

El éxito de una gestión pedagógica, en causada en la participación e inclusión de la familia y la comunidad, obligatoriamente requiere de la implantación de nuevas estrategias y redes de participación, entre ellas las asociaciones de trabajo compartido que son las comunidades de aprendizaje, con programas y proyectos que fomente los grupos de voluntariado, actividades innovadoras de aprendizaje, pero diseñados de manera concertada, contextualizada y vinculante a la realidad de estos dos agentes, aprovechando al máximo también de la infinidad de recursos tecnológicos existentes.

\section{REFERENCES}

Alanya, J., Padilla, A., y Panduro, J. (2021). Propuestas abordadas a los estilos de aprendizaje: revisión sistemática. Centro Sur, E4, 178-197. http://www.centrosureditorial.com/index.php/revista/article/view/117

Alanya Beltrán, J. E. (2017). Uso de videoconferencia y actitudes hacia la matemática en estudiantes de arquitectura de una universidad privada, año 2016. Lima: UCV

Acuña, V. (2016), "Family and school: crisis of participation in contexts of vulnerability", Revista Brasileira de Estudos Pedagógicos, 97(246), 25572. http://dx.doi.org/10.1590/S2176-6681/267830331 
Aguiar, G., Yaima, D. y Imilla, C. (2020). La participación familiar en la inclusión socioeducativa de los educandos con necesidades educativas especiales, Revista de Educación - MENDIVE, 18(1) 120-133.

Akbarul, M. (2018). Parental educational status and academic achievement of students. International Journal of Creative Research Thoughts JCRT, 6(1), 11- 20.

Álvarez, C. (2016). La mejora de la participación de la comunidad en la escuela con grupos interactivos. Revista Complutense De Educación, 28(3), 809-822, en: http://dx.doi.org/10.5209/rev_RCED.2017.v28.n3.49857

Alves, F., Gomes, A., Martins, A., y Almeida, L. da S. (2017). Cognitive performance and academic achievement: How do family and school converge? European Journal of Education and Psychology, 10(2), 49-56. https://doi.org/10.1016/J.EJEPS.2017.07.001

Andrés, S. y Giró, J. (2016a). La participación de las familias en la escuela: una cuestión compleja. Revista de Evaluación de Programas y Políticas Públicas, 7, 28-47, en: https://doi.org/10.5944/reppp.7.2016.16302

Andrés, S. y Giró, J. (2016b). El papel y la representación del profesorado en la participación de las familias en la escuela, Revista Electrónica Interuniversitaria de Formación del Profesorado, 19(1) 61-71. http://dx.doi.org/10.6018/reifop.19.1.245461.

Apaza, F. (2018). Gestión comunitaria y clima organizacional en la Institución Educativa de educación básica especial "Don José de San Martin”- Cusco [Tesis de Doctorado, Universidad César Vallejo]. https://tinyurl.com/yc26rb3r

Arostegui, I., Leire D. y Nekane, B. (2016). La participación de las familias y de otros miembros de la comunidad como estrategia de éxito en las escuelas, Revista Iberoamericana De Evaluación Educativa, 6(2), 187-200. 
Beltran, J. A., Cámaco, D., Cárdenas Palomino, F., y Velarde Vela, L. (2021). Estilos de aprendizaje de los estudiantes de arquitectura de primer ciclo del curso de matemática en tiempos de pandemia. Sinergias Educativas. http://sinergiaseducativas.mx/index.php/revista/article/view/249

Barrientos, C., Patricia, S., y Serafín, A. (2016). El Papel directivo y la promoción de la participación de las familias y la comunidad en las escuelas básica. El Caso de la Comuna de Panguipulli-Chile, REICE. Revista Iberoamericana sobre Calidad, Eficacia y Cambio en Educación, 14(3), 145-165. https://doi.org/10.15366/reice2016.14.3.008

Burgoyne, J. y Michael R. (1997). Management learning: Integrating perspectives in theory and practice, London, SAGE Publications.

Calvo, M. y Antonio, M. (2016). La Participación Familiar es un Requisito Imprescindible para una Escuela Inclusiva, Revista Latinoamericana de Educación Inclusiva, 10(1), 99-113. http://dx.doi.org/10.4067/S071873782016000100006

Cárdenas-Palomino, F., Alanya Beltran, J., Cámaco, D. D. la C., y Rojas-Zuñiga, L. (2021). Identificación de los Estilos de aprendizaje de los Estudiantes Universitarios: Revisión de Literatura. Sinergias Educativas. http://sinergiaseducativas.mx/index.php/revista/article/view/255

Chiquillo, M. y Llopis, M (2020). El grupo de WhatsApp de padres, ¿facilita o dificulta la educación de los hijos?, Edetania. Estudios y Propuestas Socioeducativas, 56 , 115-133, https://doi.org/10.46583/edetania_2019.56.50.

Córdova, N. (2018). Liderazgo directivo y participación de los padres de familia en la I.E. Secundaria “Rómulo Pedraza Pacheco”, Pacobamba 2017. [Tesis de Maestría - Universidad César Vallejo]. 
Delgado, P. (2017). Gestión comunitaria y participación de la asociación de padres de familia de la institución educativa primaria $N^{o} 3057$ de Carabayllo. [Tesis de Maestría -Universidad César Vallejo]

Díaz, M. (2015). Discursos existentes respecto a la participación de la comunidad y las familias en la gestión de la escuela. Tesis realizada en la IE 38231 Pacchahuallhua, del distrito de Independencia - Ayacucho - 2014, [Tesis de Maestría - Universidad Nacional Mayor de San Marcos]

Dove, M., Jennifer, Z., K. (2018). School Community Connectedness and Family Participation at School", World Journal of Education, 8(1), 49-57. https://doi.org/10.5430/wje.v8n1p49.

Epstein, J. y Sheldon, S. (2016). Necessary but Not Sufficient: The Role of Policy for Advancing Programs of School, Family, and Community Partnerships. The Russell Sage Foundation Journal of the Social Sciences, 2(5). 202-219. https://doi.org/10.7758/rsf.2016.2.5.10

Gaitán, A., Rodríguez, Y., y Patarroyo, L. (2016). Reflexiones sobre la participación de las familias en la gestión escolar, Hojas y Hablas, 13, 97-109.

García, P., Hernández, A., Parra, J. y Gomariz, A. (2016). Participación familiar en la etapa de educación primaria, Perfiles Educativos, 38(154), 97 - 117.

Garreta, J. (2016). Fortalezas y debilidades de la participación de las familias en la escuela, Perspectiva Educacional, 55(2), 141-157. http://dx.doi.org/10.4151/07189729-Vol.55-Iss.2-Art.395

Gobierno de Colombia (2008). Guía para el mejoramiento institucional. De la autoevaluación al plan de mejoramiento, Colombia, Ministerio de Educación Nacional.

Gobierno de Perú (2019). Perfiles de liderazgo pedagógico en escuelas primarias y su relación con el rendimiento, Perú, UMC. 
Gobierno de Perú (2014). Marco de Buen Desempeño del Directivo, Lima, Ministerio de Educación.

Gobierno de Perú (2003). Ley N. 28044, de 17 de julio, Ley General de Educación, Lima, Ministerio de Educación.

Gobierno de Perú (2005). Ley N. ${ }^{\circ} 28628$ de 25 de noviembre, Ley que regula la participación de las asociaciones de padres de familia en las instituciones educativas públicas, Lima, Ministerio de Educación.

Gómez, Y. y Vera, N. (2019). Programa de estrategias de participación social para mejorar la gestión comunitaria en la institución educativa Hermógenes Mejía Solf de la beneficencia pública de Jaén, [Tesis de MaestríaUniversidad César Vallejo].

González, N. (2019). El apoyo familiar en el proceso de formación académica en los estudiantes, Revista Científica Estudios E Investigaciones, 7, 74-75. https://doi.org/10.26885/rcei.foro.2018.74.

Holguin-Alvarez, J., Taxa, F., Tortora, E., Alanya-Beltran, J., Panduro-Ramírez, J., y Soto-Hidalgo, C. (2020). Video games and kahoot! As cognitive gamifiers in compulsory social isolation. International Journal of Advanced Trends in Computer Science and Engineering, 9(5), 86158620. https://doi.org/10.30534/ijatcse/2020/245952020

Jacay, E. (2018). La gestión educativa y la participación comunitaria en los Núcleos Educativos de Secundaria Tutorial en las regiones Pasco, Junín y Huancavelica 2018, [Tesis de Maestría- Universidad César Vallejo].

James, C. y Guzmán, E. (2016). La participación de madres y padres de familia en la escuela: un divorcio de mutuo consentimiento, Sinéctica, Revista Electrónica de Educación, 46, 1-23.

Jiménez, Y., y Kreisel, M. (2018). Participación comunitaria en educaciónreconfiguraciones de lo escolar y de la participación social, Revista 
Interuniversitaria, Vol. 30, núm. 2, pp. 223-246, en: http://dx.doi.org/10.14201/teoredu302223246.

Kankaew, K., Alanya-Beltran, J., Khamidullina, Z., Magulod, G. C., Jr, Capulso, L. B., Cabacang, G. S., Anh, V. T., Vela, L. A. P., y Pentang, J. T. (2021). Immediate program learning outcomes of information technology candidates and their introspections towards IT education relevance and global competence initiatives psychology and education. Psychology and Education, $58(2)$, 5417-5427. http://www.psychologyandeducation.net/pae/index.php/pae/article/view/29 53php/pae/article/view/2953/2618

Lavid, J. (2019). Gestión educativa y participación familiar en el Centro de Desarrollo Infantil Los Popeyes Guayaquil - Ecuador, 2018, [Tesis de Maestría-Universidad César Vallejo]

Loaiza, J. (2017) Participación de los padres de familia y el desarrollo de logros de aprendizajes de los estudiantes del VII ciclo del nivel secundaria de la Institución Educativa $N^{\circ} 16019$ San Marcos del caserío el Nogal, Jaén, 2014, [Tesis de Maestría-Universidad Nacional de Cajamarca]

Londoño, J. (2017). Papás tecnológicamente comprometidos Estrategia educativa TIC para el fortalecimiento de la participación de los padres en compromisos escolares de los niños, [Tesis de Maestría-Universidad de la Sabana].

López, L. y Ramírez, A. (2020). Acoso e inconvivencia escolar: el rol de la participación familiar en los centros educativos, Campo Abierto. Revista De Educación, 39(1), 55-77. https://doi.org/10.17398/0213-9529.39.1.55.

Madrid, R., Saracostti, M., Reininger, T., y Hernández, M. (2019). Responsabilización, obediencia y resistencia: perspectivas de docentes y padres sobre la colaboración familia-escuela, Revista Electrónica 
Interuniversitaria De Formación Del Profesorado, 22(3), 1-13, en: https://doi.org/10.6018/reifop.389801

Manayay, L. (2019). Programa de gestión estratégica en la participación de los padres de familia y comunidad en el proceso educativo de la I.E. de Nivel Primaria $N^{\circ} 10090$ del Caserio de Huarhuar - Distrito de Incahuasi, [Tesis de Maestría - Universidad Nacional Mayor de San Marcos]

Manzon, M., Miller, R., Hong, H., y Khong, L. (2015). Parent Engagement in Education, National Institute of Education, 7.

Medina, M. (2016). El proceso de Gestión Educativa en la participación de los padres de familia y tutores de los niños y niñas del primer grado sección A de la Escuela República de China, en la ciudad de Tegucigalpa, Francisco Morazán [Tesis de Doctorado- Universidad Pedagógica Nacional Francisco Morazán]

Ochoa Tataje, F. A., Panduro-Ramirez, J., Alanya-Beltran, J., y Quispe Yapu, L. B. (2021). Quality education: Perspective of the South American countries about Educational Quality. Journal of Business and Entrepreneurial Studie. https://www.journalbusinesses.com/index.php/revista/article/view/234

Ochoa, H. (2018). Participación de los padres de familia como corresponsables de la educación de sus hijos en una institución educativa del Callao. [Tesis de Maestría- Universidad San Ignacio de Loyola]

Organización de las Naciones Unidas para la Educación, la Ciencia y la Cultura (Unesco) (2020). Informe de Seguimiento de la Educación en el Mundo 2020: Inclusión y educación: Todos y todas sin excepción.

Organización de las Naciones Unidas para la Educación, la Ciencia y la Cultura (Unesco) (2004). Participación de las familias en la educación infantil Latinoamericana. Santiago, Santiago de Chile, Unesco. 
Organización para la Cooperación y el Desarrollo Económico (OCDE) (2019). Educación y competencias en la OCDE, Paris, OCDE.

Ovillo, L. (2019). Participación de los padres de familia en la gestión educativa de la institución Stella Maris. La Perla - 2019, [Tesis de Maestría Universidad César Vallejo].

Panduro-Ramírez, J., Alanya-Beltrán, J., Soto-Hidalgo, C., y Ruiz-Salazar, J. (2021). Student Assessment in the Digital Age: A Systematic Review in Latin America. Espirales Revista Multidisciplinaria de investigación, 5(36), 4866. https://doi.org/10.31876/er.v5i36.779

Payà, A., y Tormo, M. (2016). La participación educativa de las familias en una escuela pública valenciana. Un estudio cualitativo, Foro de Educación, 14(21) 227-248. http://dx.doi.org/10.14516/fde.2016.014.021.012

Perales, F., y Escobedo, M. (2016). La participación social en la educación: entre propuestas innovadoras y tradición educativa Revista Electrónica de Investigación Educativa, 18(1), 69-81.

Pereira, G. y Rivas, J. (2019). Padres y madres: actores indispensables al éxito académico de los niños, Educação Unisinos, 23(3), 471-487. https://doi.org/10.4013/edu.2019.233.06

Pérez, R. (2015). Participación de los padres de familia y la gestión escolar en la institución educativa Cristo Redentor de la comunidad de San Juan de Chiple, distrito de Callayuc, provincia de Cutervo año 2014, [Tesis de Maestría-Universidad Nacional de Cajamarca]

Quispe Yapu, L. B., Ochoa Tataje, F. A., y Panduro-Ramirez, J. (2021). Technological disparity in education: What is the situation during the pandemic? Journal of Business and Entrepreneurial Studie. https://www.journalbusinesses.com/index.php/revista/article/view/238 
Ramos, I. (2016). Participación de los padres de familia y su influencia en la gestión institucional de las instituciones educativas del nivel inicial UGEL Canchis - cusco 2015, [Tesis de Maestría-Universidad Andina Néstor Cáceres Velásquez]

Rojas, C. (2017). Propuesta de gestión para mejorar los procesos de vinculación comunidad - escuela en las instituciones de la red educativa Tailín del distrito de Sallique - Jaén, 2017, [Tesis de Maestría, Lambayeque, Universidad Nacional Pedro Ruiz Gallo].

Salimbeni, O. (2015). Escuela y comunidad. Participación comunitaria en el sistema escolar. Tendencias Pedagógicas, 17, 19-32.

Santana, A. y Reininger, T. (2017). Family involvement from a school family relationship framework.An analisis from the perspective of an administrative teamin a municipal school in Chile, Revista Internacional de Trabajo Social y Ciencias Sociales, 14, 55-74. https://doi.org/10.5944/comunitania.14.4

Simón, Cecilia, Climent Giné y Gerardo Echeita (2016). Escuela, Familia y Comunidad: Construyendo Alianzas para Promover la Inclusión, Revista Latinoamericana de Educación Inclusiva, 10(1), 25-42. http://dx.doi.org/10.4067/S0718-73782016000100003

Sorados, M. (2010). Influencia del liderazgo en la calidad de la gestión educativa, [Tesis de Maestría-Universidad Nacional Mayor de San Marcos]

Stefanski, A., Valli, L., y Jacobson, R. (2016) Beyond Involvement and Engagement: The Role of the Family in School-Community Partnerships, School Community Journal, 26(2), 135-160.

Sucari, W., Aza, P., Anaya, J., y García, J. (2020). Participación familiar en la educación escolar peruana, Revista Innova Educación, 1(1), 6-18. https://doi.org/10.35622/j.rie.2019.01.001. 
Tamariz, J. (2013). Participación de los padres de Familia en la Gestión Educativa Institucional, [Tesis de Maestría- Pontificia Universidad Católica del Perú].

Vielma, C. (2020). El Hecho Educativo desde la Participación de la Familia: Un Asunto Transdisciplinario, Revista Scientific, 5(15), 289-304. https://doi.org/10.29394/Scientific.issn.2542-2987.2020.5.15.14.289-304

Vigo, B., Dieste, B., y Julve, C. (2016). Voces sobre participación de las familias en la escuela y éxito escolar, Revista de Sociología de la Educación-RASE, 9(3) 320-333. https://doi.org/10.7203/RASE.9.3.8981

Villogas, M. (2019). Participación de los Padres de familia y la Gestión institucional de la Escuela inicial No 545 Manuel Polo Jiménez del distrito Pucusana En el año 2018, [Tesis de Maestría-Universidad César Vallejo].

Zavala, M. (2019). Participación de los padres de familia y su influencia en la gestión educativa de la Institución Educativa $N^{\circ} 14789$ Sullana, 2019, [Tesis de Maestría-Universidad César Vallejo] 